Research Article

\title{
Analysis of the Impact of High-Speed Railway on County Economic Development Based on the Synthetic Control Method: The Hubei Province in China
}

\author{
Jibo Yu, Yuanhang Zhou (D), Qian Huang, Xuemei Li, Yiwen Hou, and Xu Wang \\ School of Economics and Management, China University of Mining and Technology, Xuzhou 221116, Jiangsu, China \\ Correspondence should be addressed to Yuanhang Zhou; zhouyuanhang0039@163.com
}

Received 21 June 2021; Accepted 3 November 2021; Published 23 November 2021

Academic Editor: Isabella Torcicollo

Copyright ( 2021 Jibo Yu et al. This is an open access article distributed under the Creative Commons Attribution License, which permits unrestricted use, distribution, and reproduction in any medium, provided the original work is properly cited.

\begin{abstract}
Studying the impact of the high-speed railway (HSR) on economic growth has important practical significance and can provide a theoretical basis for government investment in infrastructure. In this study, the adoption of HSR was used for natural and rigorous exploration. Based on the panel data on counties in Hubei Province of China from 2001 to 2017, a synthetic control method was used to analyse the impact of HSR on the economic growth of county-level areas with different economic bases. The results showed that HSR had different influences on the economic development of county-level cities with different economic bases in the short term. The Wuhan-Guangzhou HSR had significantly promoted the gross domestic product (GDP) growth of Chibi City with a good economic foundation. The robustness test results revealed that the promoting effect was significant at a level of $3.7 \%$. The Hefei-Wuhan section of the Shanghai-Wuhan-Chengdu HSR had significantly inhibited the GDP growth of Hong'an County by developing on a weak economic foundation. The robustness test results revealed that the inhibitory effect was significant at the level of $2.8 \%$. However, the Hefei-Wuhan section had a stable and promoting effect on Macheng City. Based on the abovementioned conclusions, each county-level city should focus on long-term economic development, invest in HSR construction based on local conditions instead of blind expansion, make use of HSR to vigorously develop characteristic industries, and implement talent retention and introduction policies.
\end{abstract}

\section{Introduction}

The construction and improvement of transportation infrastructure has always been one of the essential conditions for promoting rapid economic and social development [1]. The improvement of a transportation infrastructure can shorten the spatiotemporal gap between cities, accelerate the flow of economic elements within the region, promote regional economic integration, and strengthen the close economic ties between regions, so that the market scale can be continuously expanded and the social division of labor is constantly refined. On October 1, 1964, Shinkansen, the first HSR in the world, opened in Japan, marking the arrival of a new era of HSR. The Shinkansen trains have driven the rapid economic development of the regions along the railways, greatly improving the economic competitiveness and employment attractiveness of surrounding cities [2]. HSR, with its advantages in speed, environmental protection, efficiency, comfort, and safety, has made many countries realize the importance of building their own HSR. Soon afterwards, a wave of HSR development in Europe followed. France, Germany, and Italy constructed HSR in succession. Compared with developed countries, China launched the building of HSR relatively late, yet its development was extremely fast. As of the end of 2019, the operating mileage of China's HSR reached 35,000 kilometers, ranking first in the world. It is estimated that by 2025 , the operating mileage of HSR will be 38,000 kilometers. By 2030, it is expected to achieve the HSR connection among provincial capitals and cities [3]. The rapidly developing, efficient, and safe HSR network with a complete layout in China has been transformed from its initial state as a pure passenger transportation system into a symbol of China's economic and cultural development. 
HSR has a profound impact on economic development. HSR can increase the development potential of cities, drive the growth of urban land prices $[4,5]$, and stimulate substantial increases in housing prices [6]. As an efficient, fast, green, and environmentally friendly means of transportation, the opening of HSR can greatly promote the improvement of traffic accessibility, reduce travel time costs $[7,8]$, promote the rapid development of the tourism industry [9], and have a significantly positive effect on the improvement of the regional environment [10]. Also, the opening of HSR can attract more opportunities for economic development, and the construction, operation, maintenance, and management of HSR require a large amount of skilled labor, which creates abundant employment opportunities. HSR has strengthened the economic ties between cities, accelerated the flow of economic elements, and contributed to continuous urban expansion. However, the economic development of central cities will inevitably cause problems. Will HSR drive the economic development of the surrounding areas to achieve regional coordinated development? With the improvement of the HSR network, more counties have been included in the layout of the construction of HSR stations and the orderly development of the county economy is a prerequisite for the coordinated development of the overall regional economy. However, compared with prefecture-level cities and provincial capital cities, county-level cities have a weaker economic foundation. The impact of the opening of HSR on the economic development of the county is uncertain, and it will bring great challenges to the development of the county. If we can develop our own superior industries under the dividends of high-speed railways, promote the agglomeration of economic factors with the attractiveness of the county itself, and produce an "agglomeration effect," they will promote the economic development of the entire county. However, in fact, due to the poor economic foundation and geographical location of more counties, the opening of HSR will exacerbate the loss of economic factors, produce a "siphon-effect," make the economic foundation weaker, and have a generally negative impact on the county's economic development.

This article aims to study the economic effects of the opening of HSR on county development. And, for counties or county-level cities with different economic bases, are there differences in economic effects? This study focuses on the counties in Hubei Province of China. The counties along the two HSRs were used as the processing group, and the counties in the province that had not yet been covered by the HSR network were used as the control group. Two counties and county-level cities with different economic bases were compared. The synthetic control method was adopted to analyse the impact of the HSR on the county's economic development and the differences in the impact. The research results of this study can provide a basis for the implementation of HSR construction plans in various county-level regions according to local conditions.

The remainder of this article is organized as follows: Section 2 is a literature review, Section 3 expounds on the influence mechanism of HSR on regional economic growth, Section 4 introduces the model and data, Section 5 focuses on empirical results, Section 6 focuses on discussion, and Section 7 ends with conclusions and policy recommendations.

\section{Literature Review}

Researchers mainly hold the following views on the relationship between HSR and regional economic development. HSR will promote, inhibit, or have no significant impact on regional economic development. Zhang reached different conclusions using two different methods; regression analysis showed that HSR will significantly boost the per capita GDP of cities along the railway line, while the grey prediction results suggested that HSR leads to a decrease in per capita GDP [11]. Wang and Nian using the DID method found that HSR has not played a significant role in regional economic development in the short term [12]. Li using the synthetic control method found that HSR will inhibit the county's total GDP [13]. Li et al. using the DID method found that HSR stimulates the regional GDP of Fujian Province but suppresses urban economic growth [14]. Tian et al. found that HSR will promote the economic development of cities along the railway line, but there is heterogeneity, which is reflected in the more prominent impact of HSR on the economic development of large cities [15]. Lin et al. found that HSR will drive an increase in investment in fixed assets, thereby promoting economic growth [16]. Ahlfeldt and Feddersen found that HSR can effectively promote the economic growth of the areas along the route in the long term [17]. Chen and Hall found that intercity HSR can significantly promote regional economic growth [18]. Nakagawa and Hatoko and Ortega et al. found that HSR promotes the economic growth of regions along the route in the short term but may inhibit economic growth in the long term $[19,20]$.

Although researchers are divided on the impact of HSR on regional economic development, there is consensus on the role of HSR in improving regional accessibility. Wang and Zhang found that the accessibility of Hefei and Hangzhou metropolitan areas has increased by more than $60 \%$ and the accessibility of Shanghai and Nanjing metropolitan areas has improved by approximately 30\% [21]. Luo et al. found that HSR greatly improves the traffic accessibility of cities along the route and also improves the traffic accessibility of nearby cities to a certain extent [22]. Su et al. found that HSR improves traffic accessibility and shortens the traffic time between cities [23]. Jiang et al. found that HSR expands the isochronous circle of the city and greatly improves the level of accessibility [24]. Blum et al. found that HSR can improve the regional accessibility between cities, connecting urban areas to form an economic belt or economic corridor [25]. Chang and Lee found that HSR improves the regional accessibility between cities, and it exerts a more obvious impact on large cities. Therefore, the construction of HSR should be encouraged to enhance regional accessibility [26]. Monzón et al. studied the Spanish HSR system and proved the positive effect of HSR on accessibility. From the perspective of the overall spatial distribution, the 
HSR can contribute to fair regional accessibility [27]. Cheng et al. explored the role of HSR in China and the European Union as a tool to enhance economic cohesion and competitiveness. In addition, the differences in the changes of different regions caused by accessibility were analysed [28].

The opening of HSR has accelerated the flow of economic elements and promoted industrial agglomeration in cities along the railway line. Zhang found that HSR will speed up the flow speed of economic elements between regions, which is conducive to the agglomeration effect of economic elements in cities along the line and promotes the formation of industrial agglomeration in cities along the line [29]. Zhang and Tao found that HSR promotes the economic agglomeration of central cities in the HSR network and economic factors will flow along the HSR line to central cities, thus forming a "siphonic effect" [30]. Zhao and Zhang found that HSR have promoted the rapid flow and frequent transfer of labor, materials, capital, and technology. Industrial agglomeration and resource diffusion have motivated the sharing of resources among the regions along the railway line [31]. Lin et al. found that HSR reduces transportation costs in areas along the line and enhances the ability of cities along the line to attract talents through time and space compression and the flow and agglomeration of factors, hence motivating industrial agglomeration [32]. Li et al. found that HSR stimulates industrial agglomeration in the western region and causes industrial diffusion in the eastern and central regions [33]. Kobayashi and Okumura found that HSR can enhance the exchange of economic factors between cities and promote the aggregation of economic factors [34]. Vickerman found that HSR promotes the concentration of economic factors in large cities and industrial agglomeration [35]. Tian et al. found that HSR can promote the agglomeration of the service industry [36].

An increasing number of studies have focused on the impact of HSR on regional economic growth, and useful results have been achieved. Empirical studies are mostly based on methods such as DID or PSM-DID [37-42]. However, the DID model needs to meet the hypothesis on common trend, and the selection of the control group is subjective and arbitrary to some extent, which will lead to hidden deviations. In this article, the synthetic control method proposed by Abadie and Gardeazabal [43] was used to analyse and categorise the counties without high-speed train stations into synthetic control areas. The weighted synthetic control areas have the same time trend as the processing group; that is, the hypothesis on common trend is met. However, the level of the weighted combination was obtained from the objective data analysis of the control group, which reduces subjectivity and arbitrariness. In recent years, the use of the synthetic control method has gradually been widening in the evaluation of policy effectiveness, such as the evaluation of the pilot property tax [44] and the low-carbon pilot policy [45]. Most of the previous studies of the impact of HSR on economic growth are based on panel data of provinces or cities [46], while panel data of county-level areas are less commonly used. In China's blueprint of the HSR network, an increasing number of county-level cities will have high-speed train stations and county-level cities are more susceptible to the "siphonic effect" than provinces or cities. The opening of HSR will have heterogeneous effects on county-level cities with different economic foundations and different geographical locations. Therefore, in this study, using the HSR in Hubei Province of China as the research object, the synthetic control method was adopted to analyse the impact of the HSR on the economic development of county-level areas with different economic bases.

\section{The Influence Mechanism of HSR on Regional Economic Growth}

HSR can influence the development of the regional economy at the macrolevel and microlevel. Based on the research of other researchers $[11,13]$, this article describes the impact mechanism of HSR on regional economic growth in Figure 1. On the macrolevel, the opening of HSR promotes the improvement of traffic accessibility, reduces traffic costs and time costs, and promotes the development of the regional economy. At the microlevel, the opening of HSR promotes the economic elements of weak economic areas to accelerate the transfer to the economic centre city and inhibits regional economic development in the short term. The opening of HSR will promote the economic elements of surrounding areas to flow into economically developed cities, forming industrial agglomeration. However, when the marginal income of the economic elements of the central city gradually decreases and the urban economic development is saturated, the economic elements and industries of the central city will spread to the surrounding areas. The opening of HSR undoubtedly speeds up the diffusion process and promotes the joint development of the central city and the surrounding cities.

3.1. Improved Traffic Accessibility. Transportation infrastructure has always been one of the necessary conditions for regional economic development. The improvement of transportation infrastructure can promote the rapid flow of economic factors among regions. After the opening of HSR, the flow speed of economic factors is accelerating, but it has a microlevel impact on the economy. At the macrolevel, the opening of HSR greatly improves the accessibility of regional transportation and promotes the reduction of inter-regional transportation costs, while greatly reducing people's time costs, thus making a span of time more integrated with economic activities and promoting regional economic development. The improvement of traffic accessibility promotes the cross-regional development of economic activities. Through the role of HSR, an economic belt is formed among regions to promote the coordinated development of industrial economy among regions.

3.2. Agglomeration Effect and Diffusion Effect. In economics, the agglomeration effect refers to central cities using their location advantages, resource advantages, and urban cityspace to attract industries and other economic factors from nearby areas, so that they have their own economic 


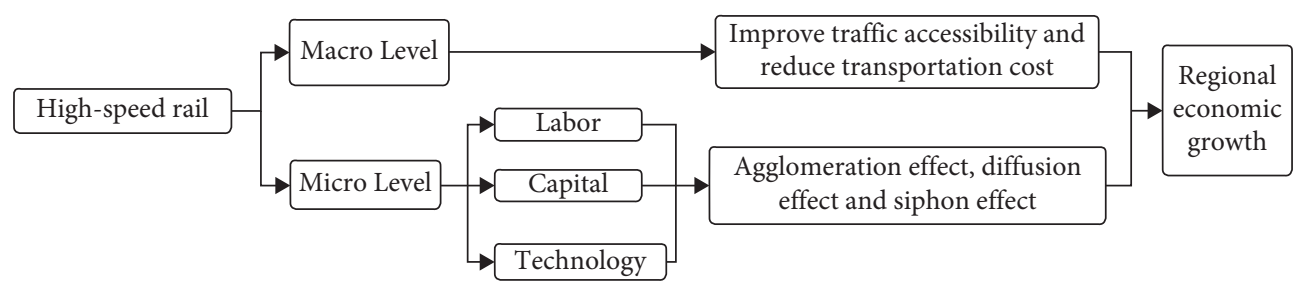

FIgURE 1: Mechanism of the impact of HSR on regional economic growth.

development benefits. On the one hand, the opening of HSR in a certain area largely provides a transit station in the surrounding areas, forming a small-scale central city, causing the agglomeration of economic factors in the nearby areas, and promoting the agglomeration of related industries. On the other hand, the opening of HSR reduces the spacetime distance between cities, improves the accessibility between cities, reduces the time cost of economic element circulation, promotes the accumulation of economic elements of manufacturers, produces economies of scale, and forms the agglomeration of related industries. The opening of HSR accelerates the flow of economic elements between cities along the line, promotes the agglomeration of related industries to cities along the line, promotes the rapid development of regional economy, undertakes industrial transfer, and promotes the optimization and upgrading of the industrial structure. The diffusion effect refers to the economic development of the central city to a certain stage, and then the effect of economic development will gradually spread to the nearby areas, driving the development of the nearby areas. With the HSR to promote the gradual formation of industrial agglomeration, economically developed areas have a large number of economic factors, which have been overly saturated, resulting in an oversupply of economic factors and promoting the reduction of economic factor cost. With the gradual reduction of cost, economic factors will gradually spread along the HSR to nearby cities and promote the diffusion of related industries to nearby areas. The opening of HSR will lead to the transfer of economic production factors to the central city, but the economic factors of land, water, mountains, and other natural resources will not be transferred to other economic production factors in the central city at a faster speed, resulting in a shortage of land, water, and other economic production factors in the central city. Thus, the relevant cost will be significantly higher than that in other areas along the line. Enterprises in central cities pursue cost minimization and profit maximization, which causes many enterprises to transfer part of their industries and factories along the HSR, in the pursuit of cost minimization. Meanwhile, the convenience of HSR will also compress the spacetime distance, making the factories relatively closer. In short, the opening of HSR brings significant agglomeration effect and diffusion effect to the cities along the line. The agglomeration effect will promote the concentration of economic factors in the central city, which makes the economically developed central city form economic factors and industrial agglomeration, increasing the rate of economic development and accelerating the problem of unbalanced economic development between regions. The diffusion effect will promote the transfer of economic factors from the economic centre to the surrounding cities along the HSR line, so that economically developed cities and economically backward cities can develop and the economic differences between them tend to converge.

3.3. Siphon Effect. The siphon effect refers to the transfer of economic factors to the central city, which leads to the weakening of the economic foundation of nearby small- and medium-sized cities and a decrease in their development potential, and has a certain inhibitory effect on regional economic development. After the opening of HSR, the setting of high-speed railway stations in small- and medium-sized cities will improve the traffic accessibility between small- and medium-sized cities and central cities, compress the spacetime distance, and reduce the time cost. However, the advantages of convenient transportation have not brought positive effects on the economic development of central cities. The economic foundation of small- and medium-sized cities is weak, and the employment attraction is poor. As a result, the economic elements, talents, and industrial chain are transferred to the economically developed central cities along the high-speed railway line. However, small- and medium-sized cities will have some leading enterprises with local characteristics according to their own regional and resource advantages. The development of these enterprises will affect the economic development of the entire city to a certain extent. However, when the HSR sets up highspeed railway stations in the region, some enterprises will choose to move their headquarters to cities with huge consumer markets. The central city with the potential for economic development will lead to the gradual loss of the leading industries to maintain regional economic development, which will limit local economic development and weaken the regional economic foundation to a certain extent.

On the macrolevel, the opening of HSR promotes the improvement of traffic accessibility, reduces the time cost, promotes more active economic activities among regions, and promotes the coordinated development of regional economy. On the microlevel, the opening of HSR makes the "siphon effect" more obvious, promotes the economic elements of small- and medium-sized cities to accelerate the transfer to the central city, weakens the economic foundation of the region, and inhibits the regional economic development in the short term. For the central city, its employment attraction and economic development potential will promote the inflow of economic factors from surrounding small- and mediumsized cities, thus forming industrial agglomeration. When the 
marginal income of the economic elements of the central city decreases and the urban economic development is saturated, the economic elements and industries of the central city will spread to the surrounding small- and medium-sized cities. The opening of the HSR undoubtedly speeds up the diffusion process and promotes the joint development of the central city and the surrounding small- and medium-sized cities, which shows the "diffusion effect."

Therefore, the diffusion effect, agglomeration effect, and siphon effect of HSR may promote or inhibit the economic development of small- and medium-sized cities. However, in small- and medium-sized cities, county-level cities occupy a large proportion, and an increasing number of county-level cities are about to open high-speed rail stations. The opening of HSR is uncertain regarding the economic growth of the county.

Based on the above analysis, this paper proposes the following hypotheses.

Hypothesis 1. The opening of HSR can promote or inhibit the economic growth of the county.

Hypothesis 2. The opening of HSR has different effects on different economic base counties.

\section{Model and Data}

\subsection{Model Setting}

$$
G_{i t}^{I}=G_{i t}^{N}+\pi_{i t} D_{i t}
$$

Assuming that there are $J+1$ county-level areas, the first area was regarded as the processing group affected by the opening of HSR and the remaining $J$ county-level areas that were not affected by the opening of HSR were regarded as the control group. The research interval is $[1, T]$, where $t=T_{0}$ is the year when the HSR was opened. $G_{i t}^{I}$ is the total GDP of the $i$-th area affected by the opening of HSR in period $t$, and $G_{i t}^{N}$ is the total GDP of the $i$-th area that was not affected by the opening of HSR in period $t$. In the year before the opening of the HSR, $t<T_{0}$ and for all areas, $D_{i t}=0$; that is, $G_{i t}^{I}=G_{i t}^{N}$; only in the processing group and after the opening of the HSR, that is, $i=1$ and $t \geq T_{0}, D_{i t}=1$; at this time, there is

$$
\pi_{i t}=G_{i t}^{I}-G_{i t}^{N} \text {, }
$$

where $\pi_{i t}$ is the economic effect of the HSR on the processing group and $G_{i t}^{I}$ is the real GDP sequence of the processing group, which is observable, but $G_{i t}^{N}$ is not observable. Therefore, it is necessary to estimate $G_{i t}^{N}$, which can be accomplished based on the factor model proposed by Abadie et al. [47]:

$$
G_{i t}^{N}=\delta_{t}+\theta_{t} Z_{i}+\lambda_{t} \mu_{i}+\varepsilon_{i t},
$$

where $\delta_{t}$ represents the time trend that affects the GDP of all $(J+1)$ county-level areas; $\theta_{t}$ is an unknown parameter, $Z_{i}$ is an observable control variable, which is a factor affecting GDP, including the proportion of each industry and investment in fixed assets; $\lambda_{t}$ is a fixed time effect, $\mu_{i}$ is a fixed regional effect, and $\varepsilon_{i t}$ is the short-term impact on each region, which is unobservable. $E\left(\varepsilon_{i t}\right)=0$.
To estimate $G_{i t}^{N}$, suppose the weight of the synthetic control area is the following $J$-dimensional column vector:

$$
w \equiv\left(w_{2}, w_{3}, \ldots, w_{J+1}\right)^{\prime}
$$

where $w_{2}$ is the weight of the second area in the synthetic processing group and all weights are nonnegative numbers, and $w_{2}+w_{3}+\cdots+w_{J+1}=1$. After weighting the outcome variable of the county-level areas in the control group,

$$
\sum_{j=2}^{J+1} w_{j} G_{j t}=\delta_{t}+\theta_{t} \sum_{j=2}^{J+1} w_{j} Z_{j}+\lambda_{t} \sum_{j=2}^{J+1} w_{j} \mu_{j}+\sum_{j=2}^{J+1} w_{j} \varepsilon_{i t} .
$$

Suppose there is a vector set $w^{*}=\left(w_{2}^{*}, \ldots, w_{J+1}^{*}\right)^{\prime}$ which satisfies

$$
\begin{aligned}
& \sum_{j=2}^{J+1} w_{j}^{*} G_{j 1}=G_{11}, \\
& \sum_{j=2}^{J+1} w_{j}^{*} G_{j 2}=G_{12}, \\
& \sum_{j=2}^{J+1} w_{j}^{*} G_{j T_{0}}=G_{1 T_{0}} \sum_{j=2}^{J+1} w_{j}^{*} Z_{j}=Z_{1} .
\end{aligned}
$$

If $\sum_{t=1}^{T_{0}} \lambda_{t}^{\prime} \lambda_{t}$ is nonsingular, then

$$
\begin{aligned}
G_{i t}^{N}-\sum_{j=2}^{J+1} w_{j}^{*} G_{j t}= & \sum_{j=2}^{J+1} w_{j}^{*} \sum_{s=1}^{T_{0}} \lambda_{t}\left(\sum_{n=1}^{T_{0}} \lambda_{n}^{\prime} \lambda_{n}\right)^{-1} \\
& \cdot \lambda_{s}^{\prime}\left(\varepsilon_{j s}-\varepsilon_{1 s}\right)-\sum_{j=2}^{J+1} w_{j}^{*}\left(\varepsilon_{j t}-\varepsilon_{1 t}\right) .
\end{aligned}
$$

Abadie et al. [47] have verified that under general conditions, the right side of equation (7) approaches 0 ; thus, when $T_{0}<t \leq T, \sum_{j=2}^{J+1} w_{j}^{*} G_{j t}$ can be used as the unbiased estimate of $G_{i t}^{N}$ to estimate $G_{i t}^{N}$. It can be found from $\pi_{i t}=$ $G_{i t}^{I}-G_{i t}^{N}$ that $G_{i t}^{I}-\sum_{j=2}^{J+1} w_{j}^{*} G_{j t}$ can be used to estimate $\pi_{i t}$ and since $G_{i t}^{I}$ and $G_{j t}$ are observable, $w_{j}^{*}$ needs to be obtained.

To select the weight $w^{*}, X_{0} W$ needs to approach $X_{1}$ as much as possible; that is, after the weighted average of the control group, the data characteristics of the synthetic control area should be as close as possible to those of the processing area. In this study, the distance between $X_{0} W$ and $X_{1}$ was selected to determine the weight $w^{*}$; that is, $\| X_{1}-X_{0}$ $W \|=\sqrt{\left(X_{1}-X_{0} W\right)^{\prime}\left(X_{1}-X_{0} W\right)}$, where $X_{0}$ and $X_{1}$ are the feature vectors of the control area and the processing area before the opening of the HSR, respectively. The weight $w^{*}$ can be determined through the minimization problem

$$
\operatorname{MSPE}=\min \frac{1}{t}\left(X_{1}-X_{0} W\right)^{\prime}\left(X_{1}-X_{0} W\right) .
$$

4.2. Data Sources and Variable Description. China's HSR is defined as a railway line with a speed of 250 kilometers per hour and above. In this study, the Hefei-Wuhan section of 
the Shanghai-Wuhan-Chengdu railway (2009.4) was designed with a speed of 250 kilometers per hour and the initial operating speed was 250 kilometers. The original designed speed of the Wuhan-Yichang section of the Shanghai-Wuhan-Chengdu railway (2012.7) was 250 kilometers per hour, but some sections are severely subsided and the speed cannot be increased and the operating speed is 200 kilometers per hour. The designed speed of the YichangWanzhou section of the Shanghai-Wuhan-Chengdu railway is only 120 to 160 kilometers per hour. The intercity railway in Hubei Province was opened late, and the synthetic control method requires economic data for a long period of time after the policy impact. Therefore, the Wuhan-Guangzhou HSR and the Hefei-Wuhan section of the Shanghai-WuhanChengdu railway were selected as the research objects in this study.

The Wuhan-Guangzhou HSR passes through Wuhan, Xianning, and Chibi in Hubei Province, while the HefeiWuhan section of the Shanghai-Wuhan-Chengdu railway passes through Macheng, Hong'an County, and Wuhan in Hubei Province. In this article, Chibi City, Macheng City, and Hong'an County which are county-level cities and counties were selected as the processing group for analysing the different impacts of HSR on the economic development of county-level areas with different economic bases. The Wuhan-Guangzhou HSR was opened on December 26, 2009, and the Hefei-Wuhan section of the ShanghaiWuhan-Chengdu railway was opened on April 1, 2009, for passenger transport. In this study, 2010 and 2009 are regarded as the time points of the policy impact of the opening of the Wuhan-Guangzhou HSR and Hefei-Wuhan section of the Shanghai-Wuhan-Chengdu railway, respectively. Chibi City, Macheng City, and Hong'an County along the railway line, where there are high-speed train stations, were selected as the processing group. The 51 county-level areas in Hubei Province that were not covered by HSR before 2018 were used as control group, as shown in Table 1.

The data were obtained from the "Hubei Provincial Statistical Yearbook" and "China's County Statistical Yearbook." However, the data of the corresponding control variables were logarithmically processed to reduce the degree of heteroscedasticity. The total GDP was used as the explained variable to measure the economic growth of the county-level areas. The control variables include general public fiscal expenditure, the year-end balance of urban and rural residents' savings, fixed asset investment, and the proportion of the added value of the secondary and tertiary industries in GDP. In addition, the total GDP in 2005 and 2008 (or 2009) was added as an additional control variable. The synth program of Stata 15.1 was used for data analysis. Table 2 shows the descriptive statistical analysis of the variables.

\section{Results}

5.1. The Impact of HSR on the Total GDP of the Processing Group. The total GDP was set as the explained variable, and the "counterfactual" state of the processing group was constructed through the data characteristics of the control group before the policy occurred, that is, the synthetic areas. By comparing the economic growth path gap between the processing group and the synthetic area after the policy occurs, the economic effect of HSR on the total GDP of Chibi City, Hong'an County, and Macheng City was explored.

Table 3 shows the weight of the county-level areas in synthetic Chibi City. A total of 4 county-level areas are engaged in the synthesis process of Chibi City, of which Guangshui City has the largest weight, 0.631 . Table 4 shows the weight of the county-level areas in the synthesis process of Hong'fan County. A total of 5 county-level areas are involved in the synthesis process of Hong'an County, among which Laohekou City has the largest weight, 0.65 . Table 5 shows the weight of the county-level areas in the synthesis process of Macheng City. There are three county-level areas participating in the synthesis process of Macheng City, among which Yingcheng City has the largest weight, 0.707.

Table 6 displays the comparison of the predictor variables of the fitting results of Chibi City, Hong'an County, and Macheng City. The true values of fiscal expenditure, fixed asset investment, the year-end balance of urban and rural residents' savings, and GDP in 2005, 2008, and 2009 in the three county-level areas are close to the predicted values. The RMSPE of the three county-level areas was within $4 \%$, which shows that the economic growth path of the synthetic county can well reflect the real economic growth path before policy implementation and proves that the synthetic control method can be used to evaluate the economic effect of highspeed railways on the processing group.

Figure 2 shows the comparison between the true value and synthetic value of GDP of Chibi City, Hong'an County, and Macheng City in the processing group. Before 2010, the GDP growth paths of Chibi City, Hong'an County, and Macheng City basically overlapped, indicating that the three county-level areas were well-fitted. After 2010, the GDP path of Chibi City increased significantly compared with that of the synthetic Chibi City and the gap between the two gradually widened until beginning to decrease in 2015. This shows that the opening of the HSR promoted the GDP growth of Chibi City in the short term. The GDP path of Hong'an County had slower growth than the path of synthetic Hong'an County, and the gap gradually widened, indicating that the opening of the Hefei-Wuhan section of the Shanghai-Wuhan-Chengdu HSR had significantly inhibited the GDP growth of Hong'an County in the short term; the GDP path of Macheng City was roughly the same as that of synthetic Macheng City, and the gap between the two was stable. This shows that the opening of the HefeiWuhan section of the Shanghai-Wuhan-Chengdu HSR had stimulated the economic growth of Macheng City to some extent.

Figure 3 shows the economic effect of HSR on the processing group. The gap in the figure represents the difference between the true value and the synthesis value of a county-level area, reflecting the economic effect of HSR on county development. From 2001 to 2009, the gap between the true value and the synthetic value of Chibi City ranged roughly between $[0.03,-0.03]$, which was reasonable. The gap increased significantly after 2010 and gradually 
TABle 1: Comparison between the processing group and the control group.

\begin{tabular}{|c|c|c|c|c|c|c|c|c|}
\hline \multirow{2}{*}{$\begin{array}{l}\text { Group } \\
\text { Processing group }\end{array}$} & \multirow{2}{*}{$\begin{array}{c}\text { Number } \\
3\end{array}$} & \multicolumn{7}{|c|}{ Name of the county-level administrative area } \\
\hline & & \multicolumn{7}{|c|}{ Chibi City, Macheng City, Hong'an County } \\
\hline Control group & 51 & $\begin{array}{c}\text { Yunxi } \\
\text { Changyang } \\
\text { Zaoyang } \\
\text { Yingcheng } \\
\text { Tuanfeng } \\
\text { Tongcheng } \\
\text { Xianfeng } \\
\text { Jiayu }\end{array}$ & $\begin{array}{l}\text { Zhushan } \\
\text { Wufeng } \\
\text { Yicheng } \\
\text { Anlu } \\
\text { Luotian } \\
\text { Chongyang } \\
\text { Laifeng } \\
\text { Xuan'en }\end{array}$ & $\begin{array}{c}\text { Zhuxi } \\
\text { Yidu } \\
\text { Jingshan } \\
\text { Gong'an } \\
\text { Yingshan } \\
\text { Tongshan } \\
\text { Hefeng }\end{array}$ & $\begin{array}{c}\text { Fang } \\
\text { Dangyang } \\
\text { Shayang } \\
\text { Jianli } \\
\text { Xishui } \\
\text { Guangshu } \\
\text { Zigui }\end{array}$ & $\begin{array}{c}\text { Danjiangkou } \\
\text { Nanzhang } \\
\text { Zhongxiang } \\
\text { Jiangling } \\
\text { Qichun } \\
\text { Lichuan } \\
\text { Laohekou }\end{array}$ & $\begin{array}{c}\text { Yuan'an } \\
\text { Gucheng } \\
\text { Xiaochang } \\
\text { Shishou } \\
\text { Huangmei } \\
\text { Jianshi } \\
\text { Yunmeng }\end{array}$ & $\begin{array}{c}\text { Xingshan } \\
\text { Baokang } \\
\text { Dawu } \\
\text { Honghu } \\
\text { Wuxue } \\
\text { Badong } \\
\text { Songzi }\end{array}$ \\
\hline
\end{tabular}

TABLE 2: Descriptive statistics of variables.

\begin{tabular}{|c|c|c|c|c|c|}
\hline Variable & Sample size & Mean & Standard deviation & Minimum & Maximum \\
\hline $\operatorname{lngdp}$ & 918 & 13.33 & 0.919 & 11.15 & 15.62 \\
\hline lnfirstprod & 884 & 12.00 & 0.766 & 10.21 & 13.82 \\
\hline secondprop & 918 & 37.93 & 10.83 & 12.28 & 67.23 \\
\hline lnsecondprod & 884 & 12.31 & 1.120 & 9.444 & 15.07 \\
\hline thirdprop & 918 & 33.70 & 6.342 & 10.22 & 55.88 \\
\hline lnthirdprod & 884 & 12.21 & 0.939 & 9.564 & 14.63 \\
\hline lnfixasset & 918 & 12.70 & 1.362 & 9.842 & 15.56 \\
\hline lnfinancialex & 918 & 11.44 & 1.092 & 8.964 & 13.63 \\
\hline lnsaving & 918 & 12.85 & 1.039 & 9.930 & 15.12 \\
\hline
\end{tabular}

lngdp represents the logarithm of GDP; firstprop, secondprop, and thirdprop, respectively, represent the ratio of the added value of the primary, second, and tertiary industry to GDP; lnfirstprod, lnsecondprod, and lnthirdprod, respectively, represent logarithm of the added value of the primary, second, and tertiary industries; lnfixasset represents the logarithm of fixed asset investment, lnfinancialex represents the logarithm of fiscal expenditure, and lnsaving represents the logarithm of the balance between urban and rural residents' savings.

TABle 3: The weight of counties and cities in synthetic Chibi City.

\begin{tabular}{lcccc}
\hline Area & Guangshui & Yidu & Yingcheng & Zhongxiang \\
\hline Weight & 0.631 & 0.032 & 0.299 & 0.038 \\
\hline
\end{tabular}

Table 4: The weight of counties and cities in synthetic Hong'an County.

\begin{tabular}{lccccc}
\hline Area & Wuxue & Laohekou & Luotian & Wufeng & Xingshan \\
\hline Weight & 0.029 & 0.65 & 0.097 & 0.154 \\
\hline
\end{tabular}

Table 5: The weight of counties and cities in synthetic Macheng City.

\begin{tabular}{lccc}
\hline Area & Yingcheng & Zhongxiang & Zhigui \\
\hline Weight & 0.707 & 0.117 & 0.176 \\
\hline
\end{tabular}

TABLE 6: Comparison of control variables.

\begin{tabular}{|c|c|c|c|c|c|c|}
\hline \multirow{2}{*}{ Variable } & \multicolumn{2}{|c|}{ Chibi City } & \multicolumn{2}{|c|}{ Hong'an County } & \multicolumn{2}{|c|}{ Macheng City } \\
\hline & True value & Synthetic value & True value & Synthetic value & True value & Synthetic value \\
\hline secondprop & 49.865 & 44.86073 & 52.345 & 45.86121 & 42.875 & 44.81798 \\
\hline thirdprop & 33.275 & 29.45442 & 32.14 & 29.2659 & 34.68 & 34.47725 \\
\hline lnfinancialex & 10.285 & 10.31316 & 10.235 & 10.11449 & 10.655 & 10.12333 \\
\hline lnfixasset & 11.745 & 11.68594 & 11.325 & 10.89898 & 11.795 & 11.6985 \\
\hline lnsaving & 11.95 & 12.30752 & 11.705 & 11.70495 & 12.08 & 12.13376 \\
\hline lngdp (2005) & 13.34 & 13.3161 & 12.56 & 12.59343 & 13.07 & 13.10484 \\
\hline lngdp (2008) & & & 13.1 & 13.10005 & 13.64 & 13.61883 \\
\hline lngdp (2009) & 13.91 & 13.92081 & & & & \\
\hline RMSPE & \multicolumn{2}{|c|}{0.01832} & \multicolumn{2}{|c|}{0.0333797} & \multicolumn{2}{|c|}{0.021254} \\
\hline
\end{tabular}



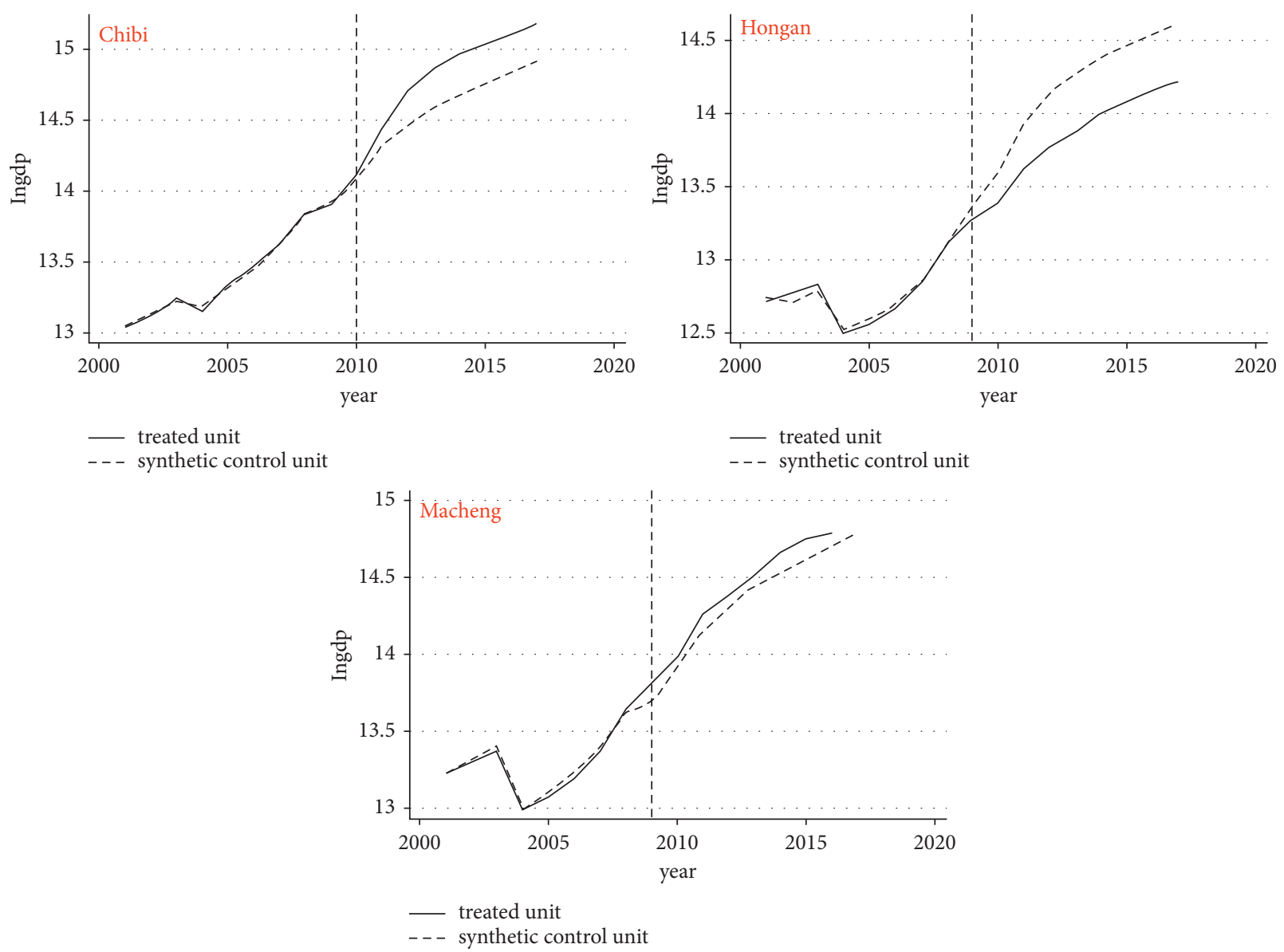

FIGURE 2: Comparison of GDP growth paths of processing groups.

decreased until 2015. The average effect from 2010 to 2017 was 0.22525 . Due to the logarithmic processing of the data, the average growth rate of the total GDP of Chibi City was $17.74 \%$, which was $4.29 \%$ higher than that of synthetic Chibi City. This shows that the opening of the Wuhan-Guangzhou HSR was indeed an important factor for boosting the GDP of Chibi City. From 2001 to 2009, before the opening of the Hefei-Wuhan section of the Shanghai-Wuhan-Chengdu railway, the gap in Hong'an County ranged between [0.06, $-0.06]$, which is reasonable. The gap decreased remarkably after 2009 (the railway was opened on April 1, 2009), showing a large negative value, and it gradually stabilized through 2015, which shows that HSR had a significant inhibitory effect on the economic growth of Hong'an County. From 2001 to 2009, the gap in Macheng City was between $[0.05,-0.05]$, which was reasonable. After 2010, the gap was positive and stable within 0.1-0.2. This shows that HSR had promoted the economic growth of Macheng City to a certain degree.

The size of the "siphonic effect" and "clustering effect" of the opening of HSR determines whether HSR can promote or suppress the county's economic development. HSR had different impacts on the economic development of counties and county-level cities with different economic foundations and geographic locations. A county-level city with a good economic foundation should have a relatively high employment population, fixed asset investment, and advantageous economic industries. However, its economic development potential was relatively high, such as the year's GDP at a relatively high level. For county-level cities with good economic foundations, regional advantages, and highquality industries, HSR was conducive to industrial agglomeration and economic factors will not be lost quickly, so that their advantageous industries can be developed. As the results of the above analysis showed, the Wuhan-Guangzhou HSR had significantly motivated the economic development of Chibi City in the short term. As a key tourist county-level city located at the junction of Hubei and Hunan, Chibi City has a good economic foundation and geographical advantage. This helps it to accept industrial transfer and attract foreign investment. Therefore, the economic effects of HSR were significantly enlarged. For impoverished counties with weak economic foundations in remote areas or in areas close to central cities, due to the lack of resources and poor employment attractiveness, the opening of HSR will accelerate the loss of economic factors, resulting in a "siphonic effect." For example, the opening of the Hefei-Wuhan section of the Shanghai-Wuhan-Chengdu HSR had significantly inhibited the economic growth of Hong'an County. Hong'an County had a weak economic foundation and was only 80 kilometers away from the provincial capital, Wuhan. Therefore, economic factors such as labor and capital will 

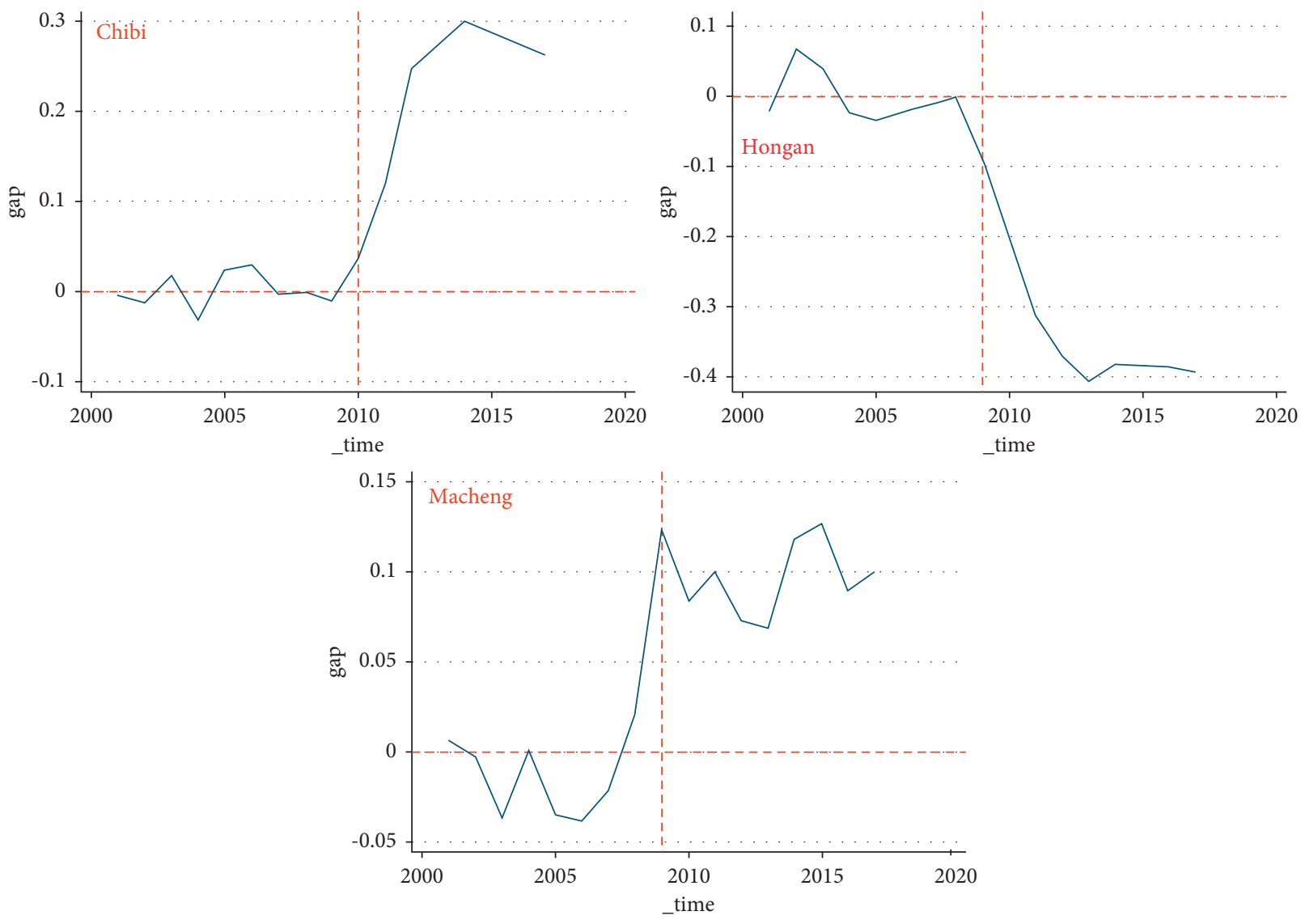

Figure 3: The economic effect of HSR on the processing group.

flow to Wuhan for development. The opening of HSR will further speed up the flow of economic factors and suppress regional economic development.

To further analyse the impact of HSR on the industrial structure of the county-level areas, an empirical analysis was performed on the impact of the Wuhan-Guangzhou HSR on the industries and the industrial structure upgrading indicator in Chibi City.

\subsection{The Impact of the HSR on the Primary Industry in Chibi} City. The added value of the primary industry was set as the explained variable, and the data characteristics of the control group before the policy impact were used to construct the "counterfactual" state of Chibi City, which was synthetic Chibi City. The gap between Chibi City and synthetic Chibi City was analysed, to explore the impact of the HSR on the added value of the primary industry in Chibi. Table 7 shows the weight combination of county-level areas in synthetic Chibi City. Synthetic Chibi City is composed of three county-level areas, and the RMSPE was 0.0305831, indicating that the economic growth path of synthetic Chibi City can fit the real economic growth path well. Figure 4 shows the comparison of the added value of the primary industry between Chibi City and synthetic Chibi City and the economic effect. This shows that the economic growth path of the synthetic area can well reflect the real economic growth path before the implementation of its policy, and the
TABLE 7: Weight combination of primary industries in synthetic Chibi City.

\begin{tabular}{lccc}
\hline Area & Xingshan County & Yidu City & Yingcheng City \\
\hline Weight & 0.118 & 0.333 & 0.549 \\
\hline
\end{tabular}

synthetic control method can be used to evaluate its policy effect. HSR plays a great role in promoting the added value of the primary industry in Chibi City.

5.3. The Impact of the HSR on the Secondary Industry in Chibi City. The added value of the secondary industry was set as the explained variable, and the data characteristics of the control group before the policy impact were used to construct the "counterfactual" state of Chibi City, which was synthetic Chibi City. The gap between Chibi City and synthetic Chibi City was analysed, to explore the impact of the HSR on the added value of the secondary industry in Chibi. Table 8 shows the weight combination of county-level areas in synthetic Chibi City. Synthetic Chibi City is composed of two county-level areas, and the RMSPE is 0.0794561 , indicating that the fit is not ideal. Figure 5 exhibits the comparison of the added value of the secondary industry between Chibi City and synthetic Chibi City, in addition to the economic effect. The gap gradually widened and became negative after the opening of the HSR. This shows that the HSR inhibits the secondary industry in Chibi. 

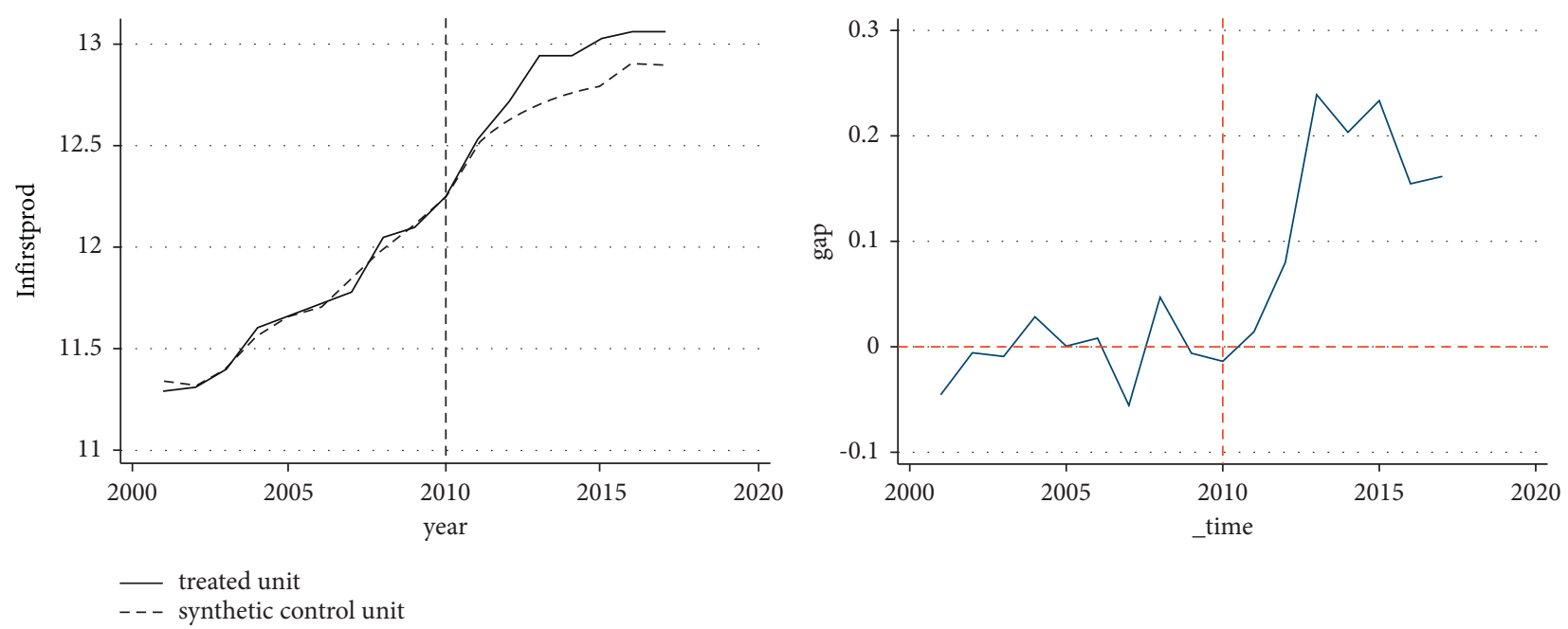

Figure 4: Comparison of economic path and economic effect of primary industry in Chibi.

TABle 8: Weight combination of secondary industries in synthetic Chibi City.

\begin{tabular}{lcc}
\hline Area & Guangshui City & Zongxiang City \\
\hline Weight & 0.313 & 0.687 \\
\hline
\end{tabular}


FIGURE 5: Comparison of economic path and economic effect of secondary industry in Chibi.

5.4. The Impact of the Opening of HSR on the Tertiary Industry in Chibi City. The added value of the tertiary industry was set as the explained variable, and the data characteristics of the control group before the policy impact were used to construct the "counterfactual" state of Chibi City, which was synthetic Chibi City. Synthetic Chibi City was composed of 19 county-level areas, and the RMSPE was 0.0698423 , indicating that the fit was not ideal. Figure 6 shows the comparison of the added value of the tertiary industry between Chibi City and synthetic Chibi City, in addition to the economic effect. The gap between the true value and the synthetic value gradually widened after the opening of the HSR, and it was a positive value. This demonstrates that the opening of the HSR had significantly promoted the added value of the tertiary industry in Chibi.
5.5. The Impact of the HSR on the Proportion of Various Industries and the Industrial Structure Upgrading Indicator in Chibi City. Figure 7 shows the changes in the proportion of the added value of each industry after the opening of the HSR in Chibi City. Before the opening of the HSR in Chibi City, the ratio of the added value of each industry to the GDP fluctuated between the average values from 2001 to 2009. Prominent changes can be found in the proportion of the three industries after the opening of the HSR. Only the proportion of the tertiary industry exhibited an upward trend, rising from $34.68 \%$ to $47.37 \%$, while the ratio of the primary industry fell from $15.6 \%$ to $11.16 \%$ and the secondary industry dropped from $49.73 \%$ to $41.47 \%$.

To better observe the role of HSR in the optimization of the industrial structure in Chibi City, the industrial structure upgrading indicator was set as ITU $=\sum_{1}^{3} i P_{i}$, 



Figure 6: Comparison of economic path and economic effect of tertiary industry in Chibi.

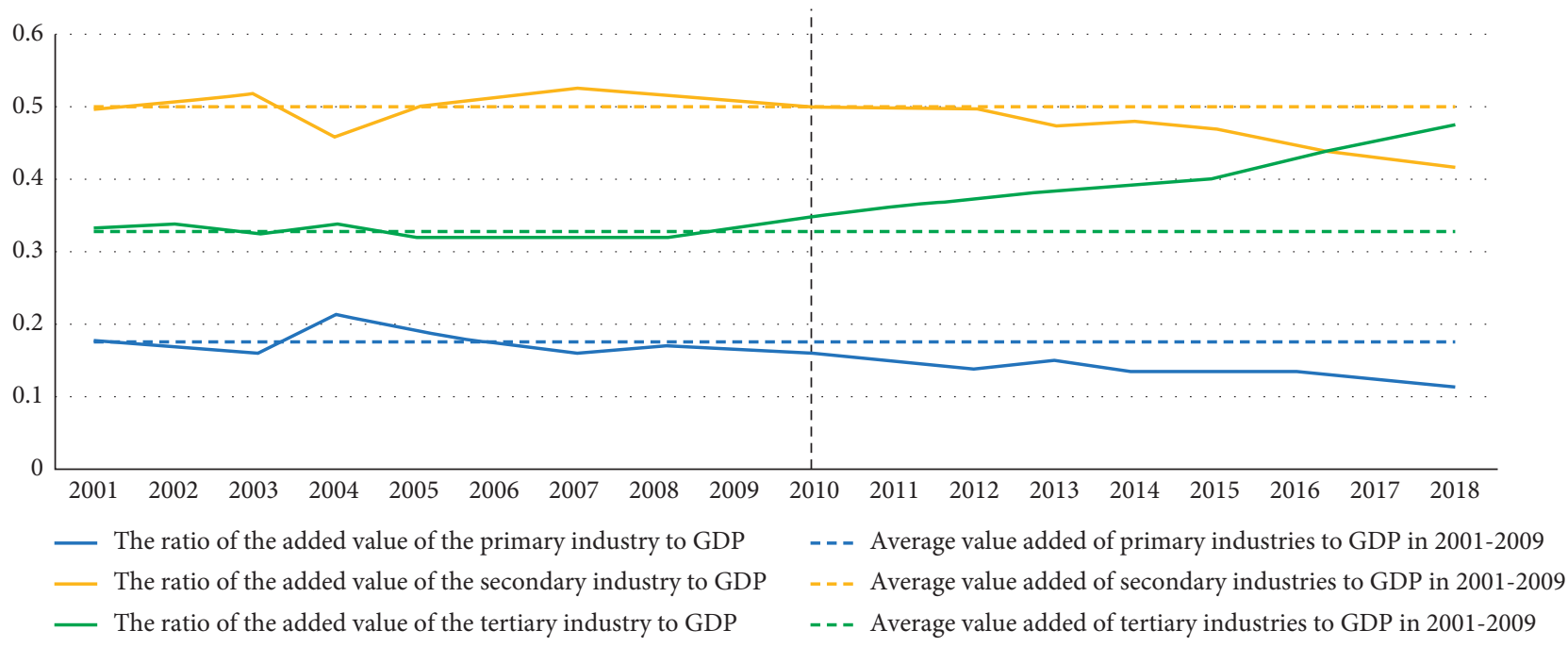

FIgURE 7: Proportion of each industry after the opening of the HSR in Chibi City.

where $P_{1}, P_{2}$, and $P_{3}$ represent the proportion of the primary, secondary, and tertiary industries, respectively. When the ITU value tends to be 3 , it shows the higher level of the industrial structure. Figure 8 shows the changes in the industrial structure upgrading indicator of Chibi City. It shows that before the opening of the HSR in Chibi City, the industrial structure upgrading indicator fluctuated between its average value from 2001 to 2009, but after the opening of the HSR, the industrial structure upgrading indicator dramatically improved, revealing that the opening of the HSR has promoted the upgrading of the industrial structure in Chibi City.

The industrial structure upgrading indicator was set as the explained variable, and the synthetic control method was used for analysis. The data characteristics of the control group before the policy impact were used to construct the "counterfactual" state of Chibi City, namely, synthetic Chibi City, and the gap between Chibi City and synthetic Chibi City was analysed, to investigate the impact of the HSR on the industrial structure upgrading indicator of Chibi City. Synthetic Chibi City was composed of five county-level areas, and the RMSPE was 0.0089092. Figure 9 shows comparison of the industrial structure upgrading indicator and economic effect in Chibi City. After the opening of the HSR, the gap significantly enlarged and was positive, indicating that the opening of the HSR greatly stimulated industrial structure upgrading in Chibi City.

5.6. Robustness Test. To verify that the economic effects of the opening of China's Wuhan-Guangzhou HSR and the Hefei-Wuhan section of the Shanghai-Wuhan-Chengdu railway on the counties along the railway line were not a contingency, time placebo tests and ranking tests were conducted on Chibi City (significant promoting effect) and Hong'an County (significant inhibitory effect), respectively, in the processing group to confirm the robustness of the results. 


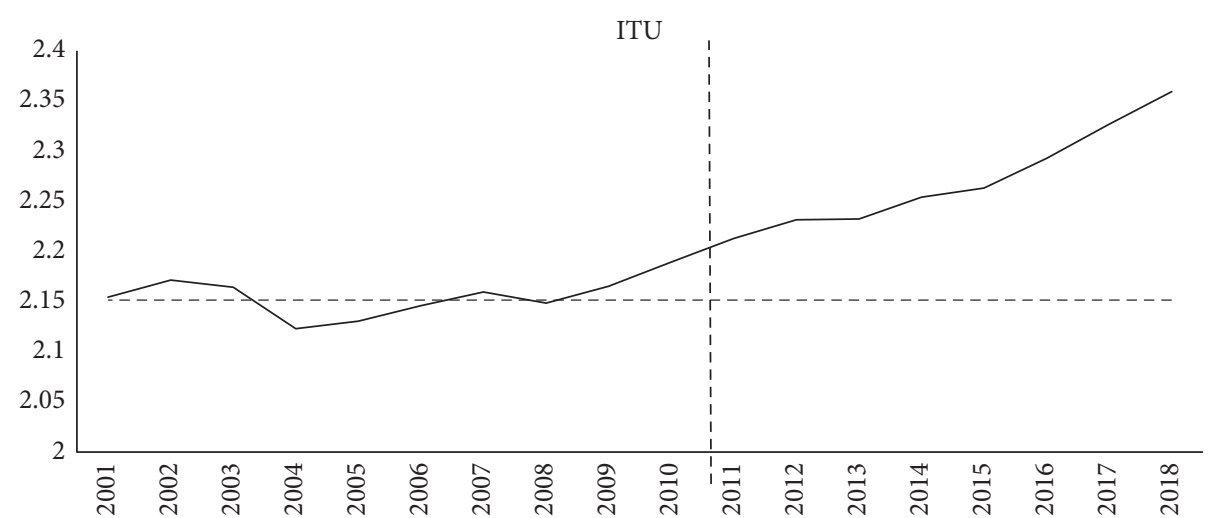

FIGURE 8: Changes in industrial structure upgrading indicator in Chibi City.
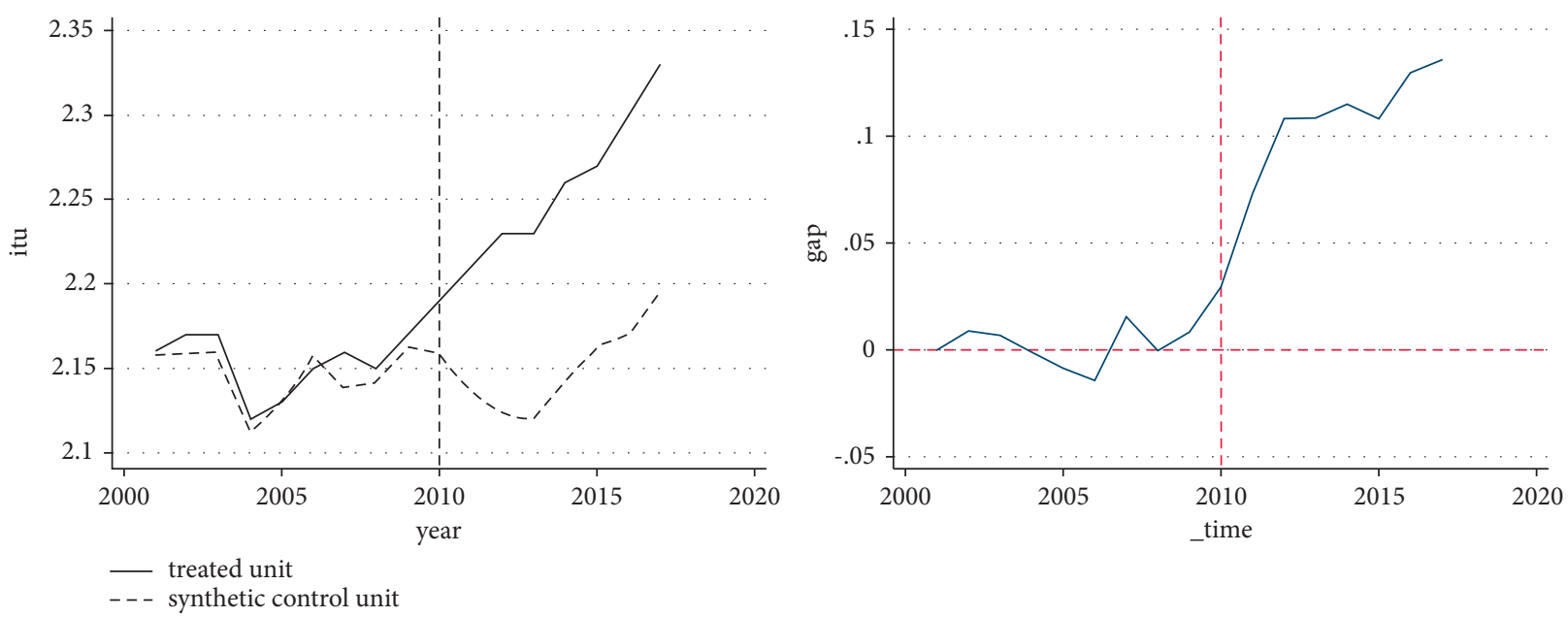

FIgure 9: Comparison of the industrial structure upgrading indicator and economic effect in Chibi City.

5.6.1. Time Placebo Test. Suppose that the WuhanGuangzhou HSR and the Hefei-Wuhan section of the Shanghai-Wuhan-Chengdu railway were opened in 2005, the synthetic control method was used again for fitting, and the fitting result is shown in Figure 10. From Figure 10(a), the total GDP of synthetic Chibi City and Chibi City from 2001 to 2018 basically overlapped, indicating that the hypothetical policy impact in 2005 had no effect on the total GDP of Chibi City. It can be found from Figure 10(b) that the gap between the true value and the synthetic value after 2005 was significantly reduced compared to the gap in 2009 when the policy impact occurred, indicating that the policy effect in 2009 is more obvious.

5.6.2. Ranking Test. According to the placebo test method proposed by Abadie et al. [47], assume that each county-level area in the control group is a processing group and the countylevel areas in the original processing group were added to the control group. Then, the synthetic control method was used to resimulate and estimate to obtain the GDP gap in each area. Next, the estimated result was compared with the GDP difference in Chibi City or Hong'an County in the empirical analysis above. If the gap in GDP between Chibi City in the processing group and synthetic Chibi City was caused by the opening of the Wuhan-Guangzhou HSR, the GDP gap of Chibi City in the processing group should be greater than the estimated result of the above hypothesis. Considering that the root mean square prediction error (RMSPE) of Chibi City is 0.0183179 , for effective comparison, the areas in the control group (51 county-level areas) with the RMSPE of the placebo test more than twice the RMSPE of the processing group were deleted and the number of the remaining areas in the control group was 26. Similarly, if the GDP gap between Hong'an County in the processing group and synthetic Hong'an County is caused by the opening of the Hefei-Wuhan section of the Shanghai-Wuhan-Chengdu railway, then the GDP gap of Hong'an County should be smaller than most of the abovementioned estimated results of the hypothesis. The RMSPE of Hong'an County is 0.0333797. For effective comparison, the areas in the control group (51 county-level areas) with the RMSPE of the placebo test more than twice the RMSPE of the processing group were deleted and the number of the remaining areas in the control group was 35 .

Figure 11(a) shows the ranking test of Chibi City. From 2001 to 2009, the GDP gap of Chibi City in the processing group was roughly the same as that of the remaining 26 areas in the control group, within $[0.1,-0.1]$. However, after the 


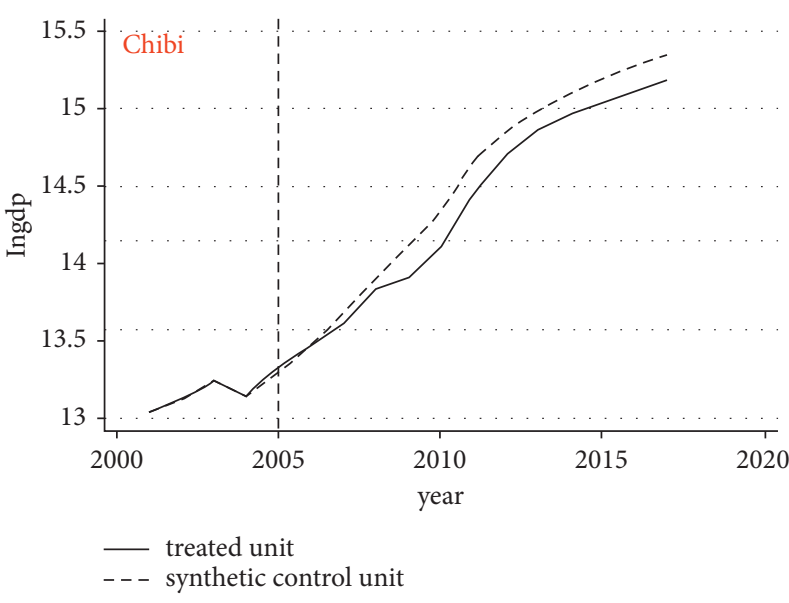

(a)

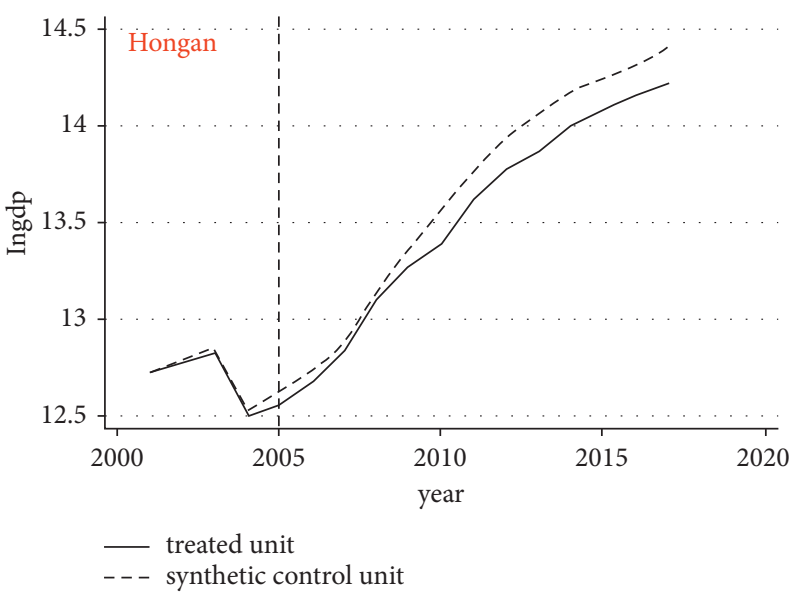

(b)

FIgURE 10: The policy impact occurred in 2005.

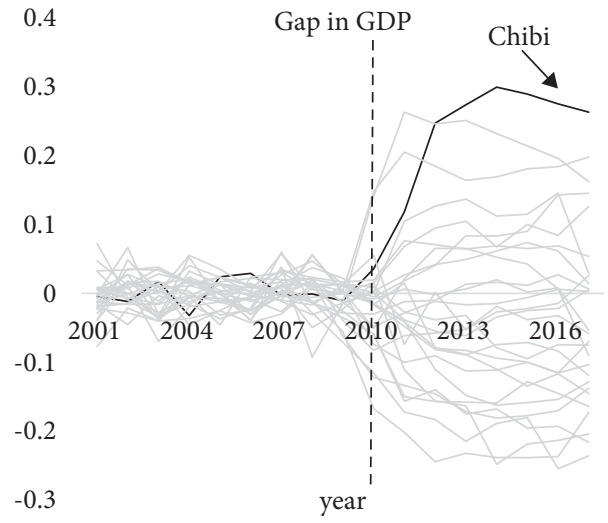

(a)

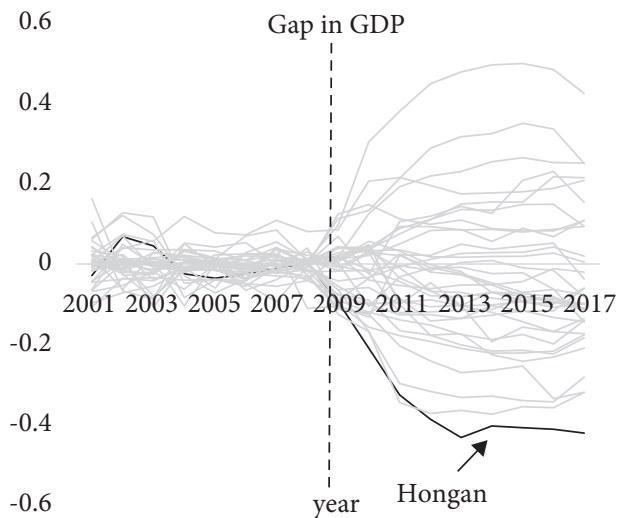

(b)

FIGURE 11: Ranking test.

HSR was opened, the GDP gap of Chibi City was significantly larger than that of the other 26 control areas. This shows that there was only a $3.7 \%(1 / 27)$ probability that there is such a large gap between Chibi City and synthetic Chibi City, indicating that the increased GDP of Chibi City caused by the opening of the Wuhan-Guangzhou HSR was significant at a level of $3.7 \%$. Figure 11 (b) shows the ranking test of Hong'an County. From 2001 to 2008, the GDP gap of Hong'an County in the processing group was roughly the same as that of the 35 control areas, within $[0.2,-0.2]$. However, after 2009, the GDP gap diagram of Hong'an County was at the bottom of the 36 areas. This reveals that the opening of the Hefei-Wuhan section has significantly inhibited the economic development of Hong'an County at a level of $2.8 \%$.

\section{Discussion}

As mentioned in the literature review, Tian et al. found that HSR will promote the economic development of cities along the railway line but accounts for heterogeneity, which is reflected in the more prominent impact of HSR on the economic development of large cities [15]. The present study verified the result showing that the opening of the two highspeed railways promoted the development of the county economy in general but showed differences in the economic development of the county with different economic foundations and different geographic locations. It also verified the hypothesis set in the influencing mechanism chapter.

The size of the "siphonic effect" and "clustering effect" of the opening of HSR determines whether HSR will promote or suppress counties' economic development in the short term. The research results showed that in the short term, HSR had significantly driven the economic development of Chibi City, a county-level city with a good economic foundation, while it had suppressed the economic growth of Hong'an County with a poor economic foundation. This had exacerbated the economic imbalance between counties. However, assessing short-term costs and benefits should not be the sole objective; rather, we should focus on long-term regional economic development. With the improved density of China's HSR network, prominent regional advantages will attract foreign investment and help the region undertake industrial transfer, so that the overall development of the region can be achieved. 
However, the opening of HSR changed the layout of the first, second, and third industrial structures in the regional economy and has a greater role in promoting the upgrading of industrial structures. After the opening of the HSR, compared with the primary and secondary industries, the proportion of the added value of the tertiary industry showed a significant upward trend. The third industry had the characteristics of unified production and consumption, wide range, small scattering, and easy to absorb labor force and plays an increasingly important role in solving employment and opening up the market. Its development had a direct impact on the level and quality of national economic development, reflecting the impact of some parts on the whole and structural optimization, which can make the whole play its maximum function. The opening of HSR promotes the development of the tertiary industry, was conducive to the overall development and progress of society, and was also conducive to improving people's living standards.

In addition, the opening of HSR shortened the time and spatial distance, so that people who work away from their hometown can quickly return to their hometown during holidays, saving time and cost, thereby increasing people's happiness. In the long run, skilled laborers who work in central cities will inevitably return to their hometown and contribute to the development of their hometown.

\section{Conclusions and Policy Recommendations}

7.1. Conclusions. The impact of HSR on economic development is a hot topic discussed at home and abroad. With the vigorous development of HSR in China, there are an increasing number of articles about the economic effects of HSR. However, in this study, the synthetic control method was used to analyse the impact of HSR on the economic growth of the county, which provides strong evidence that this method can be used in the study of "HSR economic effects." This study selects the county panel data of Hubei Province from 2001 to 2018, and the synthetic control method was used to test the impact of the WuhanGuangzhou HSR and Hefei-Wuhan section of the ShanghaiWuhan-Chengdu railway on the county's economic development.

The results showed that the Wuhan-Guangzhou HSR had significantly increased the GDP of Chibi City with a good economic foundation. It inhibits the secondary industry but promotes the primary and tertiary industries and markedly improves the industrial structure upgrading indicator. Besides, the robustness test results suggested that the GDP growth of Chibi City caused by the opening of WuhanGuangzhou HSR was significant at a level of 3.7\%. The HefeiWuhan section of the Shanghai-Wuhan-Chengdu railway had significantly inhibited the GDP growth of Hong'an County with a weak economic foundation and inhibited the growth of added value of primary, secondary, and tertiary industries. The robustness test results revealed that such inhibitory effect is significant at the level of $2.8 \%$. However, the opening of the Hefei-Wuhan section had a stable and promoting effect on Macheng. The opening of HSR had a differentiated impact on the economic development of counties or county-level cities with different economic bases. For Chibi City, which had a good economic foundation and had its own characteristic industries, such as tourism, after the opening of the HSR, the economic factors will not disappear quickly but will catch a free ride on the HSR, enjoy the HSR dividend, develop its own industries, and make the county economy develop rapidly. However, the economic foundation of Hong'an County was poor and the population was insufficient. At the same time, it was also facing the loss of labor force. The opening of HSR had accelerated the loss of county economic elements and limited economic development.

7.2. Practical Enlightenment. Based on the above conclusions, this study also had the following practical implications for county-level cities.

First, the long-term economic development of the county is the focus. We should make full use of the longterm positive effect of the HSR on the county's economy growth and should not be limited to the short-term economic effect. From the above conclusions, we see that in the short term, for counties with weak economic foundations, the opening of HSR will inhibit county economic growth. However, in the long run, the opening of HSR will bring more development opportunities and will have a comprehensive effect on regional economic development.

Second, each county-level area should implement transportation infrastructure policies based on local conditions, instead of blindly investing in HSR construction and reducing investment in other economic fields. This will lead to an uneven distribution of resources and further weaken the regional economic foundation.

Third, county-level regions should optimize the industrial layout, and HSR will facilitate the development of tourism and other service industries. All county-level regions should, based on their own historical and cultural characteristics, give play to the advantages of HSR to promote culture, tourism, and other characteristic industries.

Fourth, county-level regions should develop talent plans. The opening of HSR will accelerate the loss of economic factors in the county, the most important of which is the loss of talents. HSR will attract talent to the surrounding central cities. County-level regions should provide generous welfare policies to keep local talent and, at the same time, attract local talents working in big cities to return to their hometown for development.

\section{Data Availability}

All data generated or analysed during this study are included within this article.

\section{Conflicts of Interest}

The authors declare that there are no conflicts of interest regarding the publication of this article. 


\section{Acknowledgments}

This work was supported by the Fundamental Research Funds for the Central Universities under Project nos. 2018WA01 and 2021YCPY0112.

\section{References}

[1] X. Zhang, "Does China's transportation infrastructure promote regional economic growth-also on the spatial spillover effect of transportation infrastructure," Chinese Social Sciences, vol. 3, pp. 60-77, 2012.

[2] P. Rungskunroch, A. Jack, and S. Kaewunruen, "Socioeconomic benefits of the shinkansen network," Infrastructure, vol. 6, no. 5, p. 68, 2021.

[3] Ministry of transport, "National mid-to-long-term railway network planning," Guangxi Economy, vol. 7, p. 5, 2016.

[4] J. M. W. Low and B. K. Lee, "A data-driven analysis on the impact of high-speed rails on land prices in Taiwan," Applied Sciences, vol. 10, no. 10, p. 3357, 2020.

[5] P. Rungskunroch, Y. Yang, and S. Kaewunruen, "Does highspeed rail influence urban dynamics and land pricing?" Sustainability, vol. 12, no. 7, p. 3012, 2020.

[6] Y. Wang, X. Liu, and F. Wang, "Economic impact of the highspeed railway on housing prices in China," Sustainability, vol. 10, no. 12, p. 4799, 2018.

[7] L. Liu and M. Zhang, "The impacts of high-speed rail on regional accessibility and spatial development-updated evidence from China's mid-Yangtze river city-cluster region," Sustainability, vol. 13, no. 8, p. 4227, 2021.

[8] C. H. Wang, F. Zhen, and M. Yang, "Evaluation of the impact of Beijing-Shanghai high-speed rail on the accessibility of areas along the line," Journal of Shanxi Normal University Natural Science Edition, vol. 2, pp. 97-101, 2010.

[9] R.-C. Jou and K.-H. Chen, "The relationship between high speed rail and tourism," Sustainability, vol. 12, no. 12, p. 5103, 2020.

[10] Y. Chen, Y. Wang, and R. Hu, "Sustainability by high-speed rail: the reduction mechanisms of transportation infrastructure on haze pollution," Sustainability, vol. 12, no. 7, p. 2763, 2020.

[11] D. D. Zhang, Research on the Impact of Zhengzhou-Xi'an and Wuhan-Guangzhou High-Speed Railway on Regional Economic Development along the Line, Hunan University, Hunan, China, 2016.

[12] Y. Wang and M. Nian, "Did high-speed trains promote the development of regional economy?" Shanghai Economic Review, vol. 2, pp. 84-93, 2014.

[13] S. F. Li, High-speed Railway Construction and County's Economic Growth: A Case Study of Guangdong Province, South China University of Technology, Guangzhou, China, 2019.

[14] X. G. Li, A. M. Huang, and Y. Q. Zhang, "An impact assessment of high-speed railway on regional economic development: an empirical analysis of Fujian province based on DID mode," Modern Urban Research, vol. 4, pp. 125-132, 2017.

[15] M. Tian, T. Li, X. Ye, H. Zhao, and X. Meng, "The impact of high-speed rail on service industry agglomeration in peripheral cities," Transportation Research Part D: Transport and Environment, vol. 93, Article ID 102745, 2021.

[16] S. Lin, P. R. Dhakal, and Z. Wu, "The impact of high-speed railway on China's regional economic growth based on the perspective of regional heterogeneity of quality of place," Sustainability, vol. 13, no. 9, p. 4820, 2021.
[17] G. M. Ahlfeldt and A. Feddersen, "From periphery to core: economic adjustments to high speed rail," Documents de treball IEB, vol. 38, p. 1, 2010.

[18] C.-L. Chen and P. Hall, "The impacts of high-speed trains on British economic geography: a study of the UK's InterCity 125/225 and its effects," Journal of Transport Geography, vol. 19, no. 4, pp. 689-704, 2011.

[19] D. Nakagawa and M. Hatoko, "Reevaluation of Japanese highspeed rail construction: recent situation of the north corridor Shinkansen and its way to completion," Transport Policy, vol. 14, no. 2, pp. 150-164, 2007.

[20] E. Ortega, E. López, and A. Monzón, "Territorial cohesion impacts of high-speed rail at different planning levels," Journal of Transport Geography, vol. 24, pp. 130-141, 2012.

[21] D. G. Wang and Y. Zhang, "The impact of high-speed railway on the accessibility of the Yangtze River Delta region's metropolitans," Economic Geography, vol. 2, pp. 54-61, 2015.

[22] P. F. Luo, Y. L. Xu, and N. N. Zhang, "Research on the impact of high-speed railway on regional accessibility: a case study of Shanghai-Nanjing area," Economic Geography, vol. 24, no. 3, pp. 407-411, 2004.

[23] W. J. Su, H. T. Shi, and X. J. Wang, "The impact of BeijingShanghai high-speed rail on the main cities along the southern line of the two animals," Fudan Journal: Natural Science Edition, vol. 1, 2009.

[24] H. B. Jiang, J. G. Xu, and Y. Qi, "The impact of BeijingShanghai high-speed rail on land accessibility of regional central cities," Acta Geographica Sinica, vol. 10, 2010.

[25] U. Blum, K. E. Haynes, and C. Karlsson, "Introduction to the special issue the regional and urban effects of high-speed trains," The Annals of Regional Science, vol. 31, no. 1, pp. 1-20, 1997.

[26] J. S. Chang and J. H. Lee, "Accessibility analysis of Korean high-speed rail: a case study of the seoul metropolitan area," Transport Reviews, vol. 28, no. 1, pp. 87-103, 2008.

[27] A. Monzón, E. Ortega, and E. López, "Efficiency and spatial equity impacts of high-speed rail extensions in urban areas," Cities, vol. 30, pp. 18-30, 2013.

[28] Y.-s. Cheng, B. P. Y. Loo, and R. Vickerman, "High-speed rail networks, economic integration and regional specialisation in China and Europe," Travel Behaviour and Society, vol. 2, no. 1, pp. 1-14, 2015.

[29] X. J. Zhang, Analysis of the Impact of High-Speed Railway on the Agglomeration and Diffusion of Industries along the Line, Southwest Jiaotong University, Chengdu, China, 2016.

[30] K. Z. Zhang and D. J. Tao, "The economic distribution effect of transportation infrastructure evidence from the opening of high-speed railway," Economic Perspectives, vol. 6, pp. 62-73, 2016.

[31] D. Zhao and J. X. Zhang, "Research into spatial pattern changes of Yangtze River Delta's accessibility under the impact of high-speed railway," Resources and Environment in the Yangtze Basin, vol. 4, pp. 391-398, 2012.

[32] X. Y. Lin, Z. H. Shi, and S. Luo, "An analysis of the attractiveness of high-speed railway to talents," Journal of Beijing Jiaotong University, vol. 14, no. 3, pp. 7-16, 2015.

[33] H. C. Li, T. J. Linda, and S. X. Hu, "Agglomeration and equalization effect of high speed railway on cities in China," The Journal of Quantitative \& Technical Economics, vol. 11, pp. 127-143, 2016.

[34] K. Kobayashi and M. Okumura, "The growth of city systems with high-speed railway systems," The Annals of Regional Science, vol. 31, no. 1, pp. 39-56, 1997. 
[35] R. Vickerman, "High-speed rail in Europe: experience and issues for future development," The Annals of Regional Science, vol. 31, no. 1, pp. 21-38, 1997.

[36] M. Tian, T. Li, S. Yang, Y. Wang, and S. Fu, "The impact of high-speed rail on the service-sector agglomeration in China," Sustainability, vol. 11, no. 7, p. 2128, 2019.

[37] S. B. You and L. C. Zheng, "Evaluation of the economic stimulating effect of high-speed railway on medium sized cities along the line," Reform, vol. 296, no. 10, pp. 152-161, 2018.

[38] J. Zhang, "High-speed railway construction and county's economic development: research based on satellite lighting data," Economics: Quarterly, vol. 4, pp. 301-330, 2017.

[39] Y. Liu and Y. Li, "High-speed rails and city economic growth in China," Journal of Financial Research, vol. 33, p. 11, 2017.

[40] H. M. Ma, Z. H. Liu, and D. D. Yang, "Evaluation of the impact of high-speed railway on regional economic development: an empirical analysis of Guizhou Province based on the DID model," Journal of Guangxi University: Philosophy and Social Science, vol. 4, no. 5, pp. 88-92, 2018.

[41] D. C. Fang and M. Y. Sun, "An evaluation of the impact of high-speed rail on economic development of city clusters in the Yangtze River Delta--an empirical study based on the DID model," East China Economic Management, vol. 30, no. 2, pp. 42-47, 2016.

[42] S. X. Hu, Research on the Impact of High-Speed Railway on the Agglomeration Economy of Cities along the Line in China, Beijing Jiaotong University, Beijing, China, 2016.

[43] A. Abadie and J. Gardeazabal, "The economic costs of conflict: a case study of the Basque Country," The American Economic Review, vol. 93, no. 1, pp. 113-132, 2003.

[44] J. Y. Liu and Z. Y. Fan, "The effect evaluation of China's pilot real estate tax: a study based on synthetic control method," The Journal of World Economy, vol. 11, pp. 117-135, 2013.

[45] X. W. Lu, "Study on the effectiveness of low-carbon policy-evidence from the synthetic control methods," Soft Science, vol. 31, no. 11, pp. 98-101, 2017.

[46] Z. H. Liu and L. H. Wang, "Research on the regional economic effects and impact mechanism of transportation infrastructure-evidence from Zheng-zhou-Xi'an high-speed railway," Economic Science, vol. 2, pp. 32-46, 2017.

[47] A. Abadie, A. Diamond, and J. Hainmueller, "Synthetic control methods for comparative case studies: estimating the effect of California's Tobacco control program," Journal of the American statistical Association, vol. 105, no. 490, pp. 493$505,2010$. 